\title{
Right-to-left shunts may be not uncommon cause of TIA in Japan
}

Yohei Tateishi $^{\mathrm{a}, \mathrm{b}}$, Yasuyuki Iguchi ${ }^{\mathrm{a}}$, Kazumi Kimura ${ }^{\mathrm{a}}$, Kazuto Kobayashi ${ }^{\mathrm{a}}$, Kensaku Shibazaki $^{\mathrm{a}}$, Katsumi Eguchi ${ }^{\mathrm{b}}$

Address correspondence to:

$$
\text { Dr. Y Tateishi }
$$

${ }^{a}$ Department of Stroke Medicine, Kawasaki Medical School

577 Matsushima, Kurashiki City, Okayama 701-0192, Japan

Tel.: +81-86-462-1111 Fax: +81-86-464-1027

${ }^{b}$ First Department of Internal Medicine, Nagasaki University Graduate School of

Biomedical Sciences

1-7-1 Sakamoto, Nagasaki City, Nagasaki 852-8102, Japan

Tel.: +81-95-819-7200 Fax: +81-95-819-7215 E-mail: ytate@nagasaki-u.ac.jp

Running title: RLS and TIA

Word count: 2,067 words excluding abstract and references

Disclosure: The authors report no conflicts of interest.

Key words: patent foramen ovale, right-to-left shunt, transcranial Doppler, transesophageal echocardiography, transient ischemic attack, etiology 


\section{Abstract}

Background and purpose Although $30 \%$ to $60 \%$ of transient ischemic attacks (TIAs) have embolic sources, the etiology of the remaining TIAs is unknown. Right-to-left shunt (RLS) is one of the most important etiologies of cryptogenic stroke. The aim of this study was to determine whether RLS is related to transient ischemic attack (TIA) of unknown etiology.

Methods We performed transesophageal echocardiography (TEE) and/or transcranial Doppler (TCD) studies for consecutive TIA patients in order to detect RLS from April 2004 to December 2006. TIA patients were divided into three groups, as follows: 1) Cardioembolic TIA, with a patent cardioembolic source, 2) thrombotic TIA, with an atherothrombotic and/or lacunar mechanism, and 3) undetermined TIA, without identified cause of TIA. We compared the characteristics and presence of RLS among these three groups.

Results We enrolled 124 TIA patients (age: $67 \pm 13$ y, 80 men). There were 13 patients with Cardioembolic TIA, 25 with Thrombotic TIA, and 86 with Undetermined TIA. TEE and/or TCD were able to detect RLS in 61 of the 124 (49\%) patients. RLS was frequent in patients with Undetermined TIA compared with those in the other TIA groups $(60 \%$ in the Undetermined TIA group, $28 \%$ in 
the Thrombotic TIA group, and $15 \%$ in the Cardioembolic TIA group; $p<0.001$ ).

Smoking and previous history of TIA were frequent in the Thrombotic TIA group $(p=0.030$ and $p=0.016$, respectively)

Conclusion RLS may play an important role in the etiology of TIA of undetermined cause. 


\section{Introduction}

Transient ischemic attack (TIA) is associated with the risk of early stroke, death, and disability. ${ }^{1}$ Indeed, the appropriate strategy of treatment of patients with TIA is needed to prevent subsequent cerebral infarction. ${ }^{2,3}$ Although 30 $64 \%$ of cases of TIA have apparent embolic sources, such as carotid arterial disease or cardiac disease, ${ }^{4,5}$ there are many patients with TIA of unknown etiology. ${ }^{6}$

Paradoxical embolism is associated with cryptogenic stroke and may be a cause of stroke in a large number of patients. ${ }^{7}$ We suspect that patients with TIA of unknown or undetermined causes frequently have right-to-left shunt (RLS). The aim of this study was to determine the frequency of RLS in consecutive TIA patients, and to investigate whether TIA of undetermined cause is related to RLS.

\section{Methods}

From April 2004 to December 2006, we enrolled consecutive patients with TIA who were admitted to our hospital within 14 days of onset. TIA was defined as a sudden onset of a focal neurological deficit or amaurosis fugax, suspected 
to be of cerebrovascular origin, lasting less than 24 hours. We excluded patients who did not consent to transesophageal echocardiography (TEE) or transcranial Doppler sonography (TCD).

On admission, information on the symptoms and duration of TIA was obtained from patients or their families. We also obtained the following information about patient history and medication: 1) gender and age; 2) vascular risk factors (hypertension, diabetes mellitus, hyperlipidemia, and smoking); 3) potential cardiac sources of emboli; 4) arterial lesions (occlusive lesions of the carotid or vertebrobasilar artery able to cause neurological symptoms); and 5) previous illnesses (TIA, cerebral infarction, and ischemic heart disease). Vascular risk factors were identified as follows: 1) hypertension, use of antihypertensive agents, or systolic blood pressure $\geq 140 \mathrm{mmHg}$ or diastolic blood pressure $\geq 90 \mathrm{mmHg} ; 2$ ) diabetes mellitus, use of oral hypoglycemic agents or insulin, or glycosylated hemoglobin $\geq 6.4 \%$; 3 ) hyperlipidemia, use of antihyperlipidemic agents, or serum cholesterol level $>220 \mathrm{mg} / \mathrm{dL}$; and 4) smoking or any lifetime experience with cigarette use.

All patients were examined by 12-lead ECG, 24-hour ECG Holter monitoring, and transthoracic echocardiography to detect potential cardiac 
sources of emboli, which included atrial fibrillation, dilated cardiomyopathy, acute myocardial infarction, intraventricular thrombus, mitral valve disease, prosthetic cardiac valves, and atrial septal defect. We performed carotid duplex sonography for all patients to evaluate significant arterial lesions in the ipsilateral carotid system.

Brain magnetic resonance (MR) studies including diffusion-weighted imaging (DWI) and MR angiography were performed for all patients to investigate cerebral and cerebral arterial lesions. TCD and TEE were performed to test for the presence of RLS. Evaluation of extra- and intracranial vascular lesions was performed by carotid duplex sonography, MR angiography, CT angiography, and/or digital subtraction angiography. Diagnosis of deep venous thrombosis (DVT) and pulmonary embolism was performed using duplex sonography, scintigraphy, and/or contrast CT. Laboratory studies, including complete blood cell count, blood biochemistry, and coagulation system tests, were performed. In this study, the interpretations of all studies were blinded to the patient's clinical status. Patients and their families gave written informed consent to participate in the study, which was conducted in accordance with the Declaration of Helsinki. 


\section{MR imaging}

MR imaging studies were performed using a commercially available echo planar instrument on a 1.5-T MR unit (Signa EXCITE XL ver. 11.0; GE Healthcare, Milwaukee, WI). The neuroimaging protocol for acute stroke in our hospital included a fluid-attenuation inversion recovery (FLAIR) sequence (repetition time [TR]/echo time [TE], 8002 ms/109 ms; TI, 2000 ms; field-of-view, $24 \mathrm{~cm}$; acquisition matrix, $256 \times 224$; and section thickness, $6.0 \mathrm{~mm}$ with a 1.0-mm intersection gap), diffusion-weighted trace sequence (TR/TE, 8000 $\mathrm{ms} / 78 \mathrm{~ms}$; b values, 0 and $1000 \mathrm{~s} / \mathrm{mm}^{2}$; field of view, $24 \mathrm{~cm}$; acquisition matrix, $128 \times 192$ matrix; and $6.0 \mathrm{~mm}$ with a $1.0 \mathrm{~mm}$ intersection gap), and time-of flight MR angiography covering the circle of Willis (TR/TE, $28 \mathrm{~ms} / 6.9 \mathrm{~ms} ; 20^{\circ}$ flip angle). The acquisition time for all DWI examinations was $<1 \mathrm{~min}$. MRI findings were reviewed by experienced radiologist blinded to clinical status.

\section{TCD study}

Bilateral middle cerebral arteries were insonated from the temporal window at a depth of 50-55 mm using a $2 \mathrm{MHz}$ handheld transducer (MultiDop T; DWL, Lindau, Germany / Pioneer TC 8080; Nicolet Vascular, Madison, WI) when both middle cerebral arteries were detectable. When we could not detect middle 
cerebral artery flow from the temporal window, we detected it in the siphon of the internal carotid artery through transorbital access. A contrast study was performed to detect RLS. Contrast material was prepared by agitating $9 \mathrm{~mL}$ saline and $1 \mathrm{~mL}$ air with two syringes over a 3-way stopcock. The agitated saline was rapidly injected into a right antecubital vein during provocative maneuvers (Valsalva maneuver and coughing). Bolus injection was repeated 3 times. Doppler audio signals on TCD examination were recorded onto digital audiotape and/or hard disc. Microembolic signals were identified by typical visual appearance on the spectral display and characteristic sounds, according to standard consensus criteria. ${ }^{8}$ We validated suspected microembolic signals by "off-line" assessment without patient's clinical information.

\section{TEE study}

We performed TEE using an ultrasound unit (HDI-5000; Philips Medical System, Bothell, WA) with a 4- to 7-MHz wideband multiplane transducer for transesophageal imaging. TEE was performed in awake patients using lidocaine gel for local pharyngeal anesthesia. TEE studies included imaging of all valves and cardiac chambers. Gain settings were adjusted to distinguish background noise and spontaneous echocardiographic contrast. We circumferentially 
assessed the left atrial cavity and left atrial appendage to locate fixed or mobile echo-dense masses that could be clearly differentiated from the cardiac wall. A contrast study was performed to detect patent foramen ovale (PFO). PFO was diagnosed when at least 3 microbubbles were identified in the left atrium within 3 cardiac cycles from the right atrium. ${ }^{9}$ Atrial septal aneurysm was diagnosed when the atrial septum protruded into the left or right atrium or both for at least $15 \mathrm{~mm}$. We observed all segments of the thoracic aorta, including the descending aorta, aortic arch, and ascending aorta, to detect aortic plaque. Complicated aortic lesions were defined as those having increased intimal thickness for at least $4 \mathrm{~mm}$ in the ascending aorta or the arch of the aorta. The examinations were recorded on super VHS videotapes and reviewed by three experienced sonographers blind to clinical data.

\section{Analysis}

First, we divided the patients with TIA into three groups: Cardioembolic TIA, Thrombotic TIA, and Undetermined TIA. If TIA patients had an obvious source of emboli, such as atrial fibrillation, a mechanical prosthetic valve, dilated cardiomyopathy, left ventricular thrombus, or an akinetic left ventricular segment, they were included in the Cardioembolic TIA group. Those in the thrombotic TIA 
group had a stenotic lesion $(\geq 50 \%)$ in the relevant carotid or cerebral artery (atherothrombotic TIA), or ischemic lesions with a diameter of less than $1.5 \mathrm{~cm}$ in the territory of the basal ganglia, thalamus, or brainstem on MRI without any embolic source (lacunar TIA). The remaining patients were defined as those with TIA of "undetermined etiology", and formed the Undetermined TIA group. Patients who had two or more embolic sources were categorized in the Undetermined TIA group. We compared the background, duration of symptoms, D-dimer level, presence of source of emboli, such as RLS, atrial septal aneurysm, and complicated aortic lesion, and DVT among the three groups. We then divided the TIA patients with Undetermined TIA into two subgroups: 1) a HDM subgroup of patients with hypertension and/or diabetes mellitus, and a 2) non-HDM subgroup of patients without hypertension and diabetes mellitus. We compared clinical background and the presence of RLS between the non-HDM and HDM subgroups.

All values are the mean \pm standard deviation $(S D)$ or proportions of each group. Statistical comparison of characteristics among the three groups was performed using one-way factorial analysis of variance, such as the chi-square test, Fisher's exact test, Mann-Whitney's $U$ test, and Kruskal-Wallis test, with 
probability values $(p)<0.05$ considered significant.

\section{Results}

One hundred twenty-nine patients with TIA were admitted to our hospital from April 2004 to December 2006. We excluded five patients who did not consent to undergo TEE or TCD. Thus, 124 patients (age: $67 \pm 13$ y, 80 men) were enrolled in the present study. They were divided into three groups: the Cardioembolic TIA group of 13 patients, the Thrombotic TIA group of 25 patients, and the Undetermined TIA group of 86 patients. We performed TCD in 101 patients (81\%) and TEE in 103 patients (83\%). Eighty-one patients (65\%) underwent both TCD and TEE.

Table 1 shows the clinical background of the subjects of the three groups, and Figure 1 the prevalence of RLS. Cardioembolic TIA occurred in 10 patients with atrial fibrillation, one with a prosthetic aortic valve, one with a left ventricular thrombus, and one with dilated cardiomyopathy. Thrombotic TIA occurred in 16 patients with atherothrombotic TIA and 9 patients with lacunar TIA.

RLS was detected in 61 of 124 (49\%) patients. Fifty-two patients $(60 \%)$ in the Undetermined TIA group had RLS, along with 2 of 13 (15\%) patients in the 
Cardioembolic TIA group and 7 of 25 (28\%) patients in the Thrombotic TIA group $(p<0.001)$. A total of $72 \%$ of patients in the Thrombotic TIA group smoked. Recurrent TIA was found in 7 of $25(28 \%)$ patients in the Thrombotic TIA group. Overall, smoking and prior history of TIA were frequently observed in the Thrombotic TIA group compared with the two other groups $(p=0.030$ and $p=$ 0.016, respectively). However, there were no significant differences in other clinical characteristics among the three groups. DWI demonstrated ischemic lesions in 32 of 124 patients (26\%). The mean time from onset to MRI study was $3.3 \pm 4.3$ days. DWI revealed lesions in 3 of 13 patients $(23 \%)$ in the Cardioembolic TIA group, 15 of 25 patients (60\%) in the Thrombotic TIA group, and 14 of 86 patients $(16 \%)$ in the Undetermined TIA group $(p<0.001)$.

Table 2 shows clinical characteristics of the non-HDM and HDM subgroups. Patients in the non-HDM subgroup were younger and less likely to have vascular risk factors than those in the HDM subgroup. RLS was more frequently observed in the non-HDM subgroup than in the HDM subgroup (76\% vs $53 \%, p=0.038$ ) (Figure 2 ). The previous stroke was less in the non-HDM subgroup than in the HDM subgroup $(p=0.046)$. 


\section{Discussion}

In our study, about half of patients with TIA had RLS, and RLS was particularly frequent in patients with TIA of undetermined cause. In addition, TIA patients with neither hypertension nor diabetes mellitus frequently had RLS.

The frequency of PFO ranged from $10 \%$ to $44 \%$ for all subtypes of ischemic stroke, $31 \%$ to $77 \%$ for cryptogenic stroke, $4 \%$ to $25 \%$ for stroke of known cause, and $3 \%$ to $22 \%$ for control subjects. ${ }^{10} \mathrm{~A}$ retrospective Western study found that TEE could detect PFO in 12 of 59 (20\%) TIA patients. ${ }^{11}$ The frequency of RLS in our series was higher than in that study because most TIAs in the Western study has been caused by atherothrombotic carotid lesions. ${ }^{12}$

In our study, patients in the Undetermined TIA group frequently had RLS. This finding is consistent with the reports of Yeung et al. and Cerrato et al., which noted that RLS was prevalent in all subtypes of stroke. ${ }^{13,14}$ Some causes of undetermined TIA may include paradoxical brain ischemia detectable on DWI. When patients in the Undetermined TIA group were divided into two subgroups, the non-HDM subgroup with neither hypertension nor diabetes mellitus and the HDM subgroup with hypertension and/or diabetes mellitus, RLS was frequently observed in the non-HDM subgroup. We previously reported that RLS was 
strongly associated with lacunar stroke in patients without hypertension and diabetes mellitus. ${ }^{15}$ We therefore believe that RLS may be one of the causes of TIA in patients with neither hypertension nor diabetes mellitus.

There are some limitations to the present study. First, TEE was performed on selected patients. The rate of detection of RLS in TCD study might be inferior to that on TEE study, because the sensitivity of TCD study in detecting RLS is less than that of TEE study. ${ }^{16}$ Second, we were unable to perform peripheral venous ultrasound to detect DVT in all patients.

In conclusion, the prevalence of RLS was remarkably high in patients of the Undetermined TIA group, and RLS was particularly common in patients with Undetermined TIA with neither hypertension nor diabetes mellitus. RLS may thus be one of the mechanisms of TIA of undetermined cause. 


\section{Reference}

[1] Daffertshofer M, Mielke O, Pullwitt A, Felsenstein M, Hennerici M. Transient ischemic attacks are more than "ministrokes". Stroke 2004;35:2453-8.

[2] Rothwell PM, Warlow CP. Timing of TIAs preceding stroke: time window for prevention is very short. Neurology 2005;64:817-20.

[3] Giles MF, Flossman E, Rothwell PM. Patient behavior immediately after transient ischemic attack according to clinical characteristics, perception of the event, and predicted risk of stroke. Stroke 2006;37:1254-60.

[4] Kimura K, Minematsu K, Yasaka M, Wada K, Yamaguchi T. The duration of symptoms in transient ischemic attack. Neurology 1999;52:976-80.

[5] Weimar C, Kraywinkel K, Rodl J, Hippe A, Harms L, Kloth A, Diener HC. Etiology, duration, and prognosis of transient ischemic attacks: an analysis from the German Stroke Data Bank. Arch Neurol 2002;59:1584-8.

[6] Inatomi Y, Kimura K, Yonehara T, Fujioka S, Uchino M. DWI abnormalities and clinical characteristics in TIA patients. Neurology $2004 ; 62: 376-80$.

[7] Lechat P, Mas JL, Lascault G, Loron P, Theard M, Klimczac M, Drobinski G, Thomas D, Grosgogeat Y. Prevalence of patent foramen ovale in patients with stroke. N Engl J Med 1988;318:1148-52. 
[8] Ringelstein EB, Droste DW, Babikian VL, Evans DH, Grosset DG, Kaps

M, Markus HS, Russell D, Siebler M. Consensus on microembolus detection by TCD. International Consensus Group on Microembolus Detection. Stroke 1998;29:725-9.

[9] Cabanes L, Coste J, Derumeaux G, Jeanrenaud X, Lamy C, Zuber M, Mas JL. Interobserver and intraobserver variability in detection of patent foramen ovale and atrial septal aneurysm with transesophageal echocardiography. J Am Soc Echocardiogr 2002;15:441-6.

[10] Overell JR, Bone I, Lees KR. Interatrial septal abnormalities and stroke: a meta-analysis of case-control studies. Neurology 2000;55:1172-9.

[11] Dawn B, Hasnie AM, Calzada N, Longaker RA, Stoddard MF. Transesophageal echocardiography impacts management and evaluation of patients with stroke, transient ischemic attack, or peripheral embolism. Echocardiography 2006;23:202-7.

[12] Feinberg WM, Albers GW, Barnett HJ, Biller J, Caplan LR, Carter LP, Hart RG, Hobson RW, 2nd, Kronmal RA, Moore WS, et al. Guidelines for the management of transient ischemic attacks. From the Ad Hoc Committee on Guidelines for the Management of Transient Ischemic Attacks of the Stroke 
Council of the American Heart Association. Circulation 1994;89:2950-65.

[13] Yeung M, Khan KA, Shuaib A. Transcranial Doppler ultrasonography in the detection of venous to arterial shunting in acute stroke and transient ischaemic attacks. J Neurol Neurosurg Psychiatry 1996;61:445-9.

[14] Cerrato P, Imperiale D, Priano L, Mangiardi L, Morello M, Marson AM, Carra F, Barberis G, Bergamasco B. Transoesophageal echocardiography in patients without arterial and major cardiac sources of embolism: difference between stroke subtypes. Cerebrovasc Dis 2002;13:174-83.

[15] Ueno Y, Kimura K, Iguchi Y, Shibazaki K, Inoue T, Urabe T. Right-to-left shunt and lacunar stroke in patients without hypertension and diabetes. Neurology 2007;68:528-31.

[16] Job FP, Ringelstein EB, Grafen Y, Flachskampf FA, Doherty C, Stockmanns A, Hanrath P. Comparison of transcranial contrast Doppler sonography and transesophageal contrast echocardiography for the detection of patent foramen ovale in young stroke patients. Am J Cardiol 1994;74:381-4. 


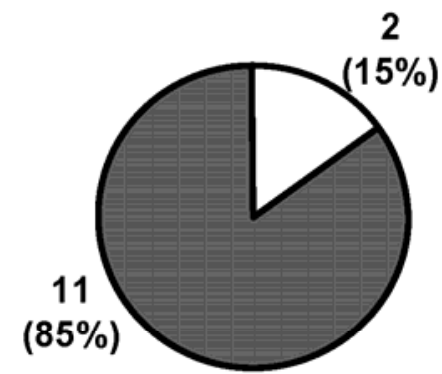

Cardioembolic TIA

group

$n=13$

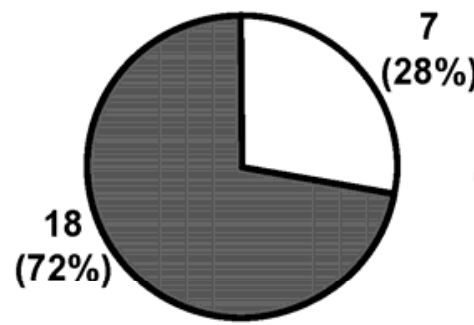

Thrombotic TIA

group

$n=25$

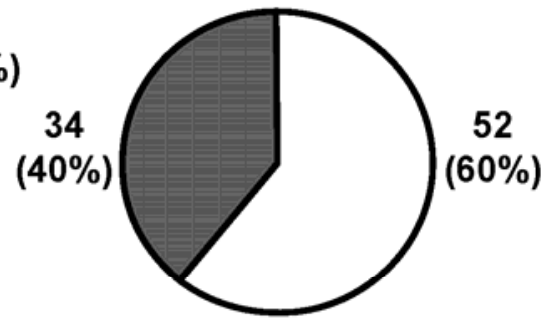

Undetermined TIA group $n=86$ $\square$ Positive RLS

Figure1. Proportion of RLS in patients with Cardioembolic TIA, Thrombotic TIA and Undetermined TIA. RLS is found in 2 of $13(15 \%)$ patients with Cardioembolic TIA, 7 of 25 (28\%) in Thrombotic TIA and 52 of $86(60 \%)$ with Undetermined TIA, respectively $(p<0.001)$. 


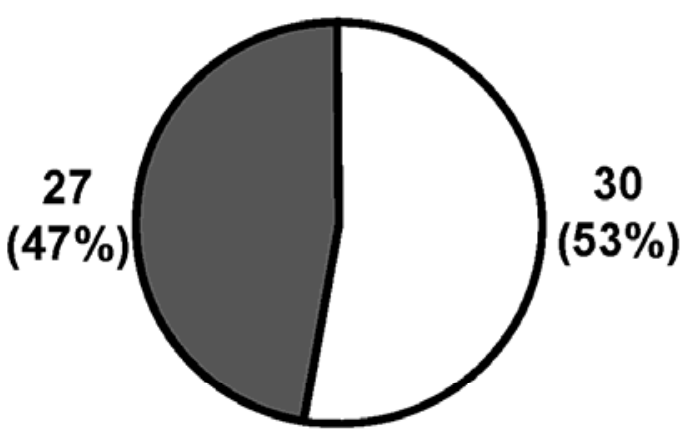

HDM subgroup

$$
\mathrm{n}=\mathbf{5 7}
$$

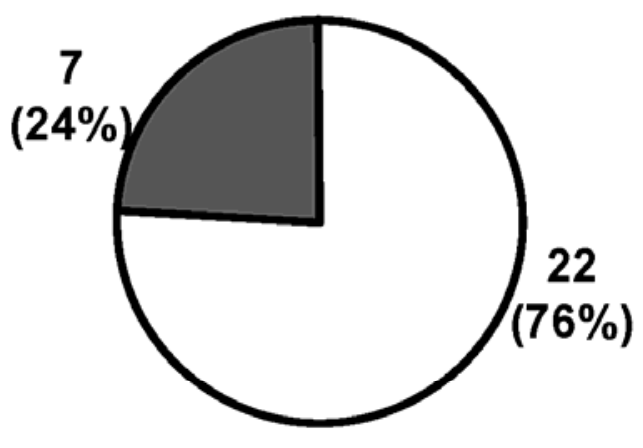

non-HDM subgroup

$$
\mathrm{n}=\mathbf{2 9}
$$

\section{$\square$ Positive RLS $\square$ Negative RLS}

Figure 2. Proportion of RLS between HDM group (patients with hypertension and/or diabetes mellitus) and non-HDM group (patients without hypertension and diabetes mellitus). RLS is observed in 30 of 57 patients of HDM group and in 22 of 29 patients of non-HDM group (53\% vs 76\%, p $=0.038)$. 
Table 1.

Clinical backgrounds of TIA patients

\begin{tabular}{|c|c|c|c|c|c|c|c|}
\hline \multirow{3}{*}{$\begin{array}{l}\text { Variables } \\
\text { Age, y, mean (SD) }\end{array}$} & \multirow{2}{*}{\multicolumn{2}{|c|}{$\begin{array}{c}\text { Cardioembolic TIA } \\
\text { group } \\
n=13 \\
\end{array}$}} & \multirow{2}{*}{\multicolumn{2}{|c|}{$\begin{array}{c}\begin{array}{c}\text { Thrombotic TIA } \\
\text { group }\end{array} \\
n=25 \\
\end{array}$}} & \multirow{2}{*}{\multicolumn{2}{|c|}{$\begin{array}{c}\text { Undetermined TIA } \\
\text { group } \\
\mathrm{n}=86 \\
\end{array}$}} & \multirow{3}{*}{$\frac{P}{0.071}$} \\
\hline & & & & & & & \\
\hline & 69 & $(13)$ & 63 & (13) & 68 & $(12)$ & \\
\hline Male, n (\%) & 9 & $(69)$ & 20 & $(80)$ & 51 & $(59)$ & 0.155 \\
\hline Duration of symptom, min, mean (SD) & 508 & $(617)$ & 152 & (323) & 204 & $(373)$ & 0.063 \\
\hline \multicolumn{8}{|l|}{ Vascular risk factors, n (\%) } \\
\hline Hypertension & 8 & $(62)$ & 19 & $(76)$ & 51 & $(59)$ & 0.316 \\
\hline Diabetes Mellitus & 2 & (15) & 10 & (40) & 19 & $(22)$ & 0.136 \\
\hline Hyperlipidemia & 4 & (31) & 16 & $(64)$ & 39 & $(45)$ & 0.116 \\
\hline Smoking & 6 & (46) & 18 & $(72)$ & 36 & $(42)$ & 0.030 \\
\hline \multicolumn{8}{|l|}{ Past history, n (\%) } \\
\hline TIA & 0 & $(0)$ & 7 & $(28)$ & 8 & (9) & 0.016 \\
\hline Stroke & 1 & (8) & 2 & $(8)$ & 12 & (14) & 0.813 \\
\hline Ischemic heart disease & 1 & $(8)$ & 4 & (16) & 7 & $(8)$ & 0.491 \\
\hline Atrial fibrillation, n (\%) & 10 & $(77)$ & 0 & $(0)$ & 2 & $(2)$ & $<0.001$ \\
\hline Arterial lesion ${ }^{\star}, \mathrm{n}(\%)$ & 0 & $(0)$ & 16 & (64) & 5 & (6) & $<0.001$ \\
\hline Right-to-left shunt, n (\%) & 2 & (15) & 7 & (28) & 52 & $(60)$ & $<0.001$ \\
\hline Atrial septal aneurysm ${ }^{\dagger}, \mathrm{n}(\%)$ & $1 / 12$ & (8) & $1 / 19$ & $(5)$ & $11 / 72$ & (15) & 0.454 \\
\hline Aortic complicated lesion ${ }^{\dagger}, \mathrm{n}(\%)$ & $3 / 12$ & (25) & $8 / 19$ & $(42)$ & $24 / 72$ & (33) & 0.608 \\
\hline Ischemic lesion on DW, n (\%) & 3 & (23) & 15 & $(60)$ & 14 & (16) & $<0.001$ \\
\hline Deep venous thrombosis ${ }^{\ddagger}, \mathrm{n}(\%)$ & $1 / 7$ & (14) & $0 / 10$ & $(0)$ & $5 / 58$ & (9) & 0.533 \\
\hline D-dimer, $\mu \mathrm{g} / \mathrm{dl}$, mean $(\mathrm{SD})$ & 1.2 & $(1.1)$ & 1.0 & $(1.3)$ & 1.5 & $(5.2)$ & 0.736 \\
\hline
\end{tabular}

SD; standard deviation, TIA; transient ischemic attack, DW; diffusion weighted image

* "Arterial lesion" is the occlusive lesion of carotid or vertebrobasilar artery which is able to cause the neurological symptom.

† TEEs were performed in 12 patients of Cardioembolic TIA, 19 of Thrombotic TIA and 72 of Undetermined TIA.

₹ In order to detect the deep venous thrombosis, duplex sonography, scintigraphy, and/or contrast CT were studied in 7 patients of Cardioembolic TIA, 10 of Thrombotic TIA and 58 of Undetermined TIA. 


\section{Table 2.}

Clinical backgrounds of TIA patients with the non-HDM subgroup and HDM subgroup

\begin{tabular}{|c|c|c|c|c|c|}
\hline \multirow{3}{*}{$\begin{array}{l}\text { Variables } \\
\text { Age, y, mean (SD) }\end{array}$} & \multirow{2}{*}{\multicolumn{2}{|c|}{$\begin{array}{c}\text { HDM subgroup } \\
n=57\end{array}$}} & \multirow{2}{*}{\multicolumn{2}{|c|}{$\begin{array}{c}\text { non-HDM subgroup } \\
\mathrm{n}=29\end{array}$}} & \multirow{3}{*}{$\frac{P}{0.062}$} \\
\hline & & & & & \\
\hline & 71 & $(10)$ & 64 & $(15)$ & \\
\hline Male, $n(\%)$ & 34 & $(60)$ & 17 & $(59)$ & 0.927 \\
\hline Duration of symptom, min, mean (SD) & 202 & $(379)$ & 206 & (369) & 0.364 \\
\hline \multicolumn{6}{|l|}{ Vascular risk factors, $\mathrm{n}(\%)$} \\
\hline Hypertension & 51 & (89) & 0 & $(0)$ & \\
\hline Diabetes Mellitus & 19 & (33) & 0 & $(0)$ & \\
\hline Hyperlipidemia & 31 & $(54)$ & 8 & (28) & 0.019 \\
\hline Smoking & 24 & (42) & 12 & (41) & 0.949 \\
\hline \multicolumn{6}{|l|}{ Past history, n (\%) } \\
\hline TIA & 5 & (9) & 3 & $(10)$ & 0.813 \\
\hline Stroke & 11 & (19) & 1 & (3) & 0.046 \\
\hline Ischemic heart disease & 7 & (12) & 0 & $(0)$ & 0.050 \\
\hline Atrial fibrillation, $\mathrm{n}(\%)$ & 2 & (4) & 0 & $(0)$ & 0.310 \\
\hline Arterial lesion, $\mathrm{n}(\%)$ & 4 & $(7)$ & 1 & (3) & 0.506 \\
\hline Right-to-left shunt, n (\%) & 30 & (53) & 22 & (76) & 0.038 \\
\hline Atrial septal aneurysm ${ }^{\dagger}, \mathrm{n}(\%)$ & $7 / 45$ & (16) & $4 / 27$ & (15) & 0.528 \\
\hline Aortic complicated lesion ${ }^{\dagger}, \mathrm{n}(\%)$ & $19 / 45$ & $(42)$ & $5 / 27$ & (19) & 0.082 \\
\hline Ischemic lesion on DW, $\mathrm{n}(\%)$ & 12 & (21) & 2 & $(7)$ & 0.095 \\
\hline Deep venous thrombosis ${ }^{\ddagger}, \mathrm{n}(\%)$ & $4 / 34$ & $(12)$ & $1 / 24$ & (4) & 0.314 \\
\hline D-dimer, $\mu \mathrm{g} / \mathrm{dl}$, mean (SD) & 1.9 & $(6.4)$ & 0.7 & $(0.3)$ & 0.843 \\
\hline
\end{tabular}

SD; standard deviation, TIA; transient ischemic attack

* "Arterial lesion" is the occlusive lesion of carotid or vertebrobasilar artery which is able to cause the neurological symptom.

$\dagger$ TEEs were performed in 45 patients of HDM subgroup and 27 of non-HDM subgroup.

₹ In order to detect the deep venous thrombosis, duplex sonography, scintigraphy, and/or contrast CT were studied in 34 of HDM subgroup and 24 of non-HDM subgroup. 